PROCEEDINGS OF THE

AMERICAN MATHEMATICAL SOCIETY

Volume 129, Number 6, Pages 1631-1634

S 0002-9939(00)05755-5

Article electronically published on October 31, 2000

\title{
ON THE LYUBEZNIK NUMBERS OF A LOCAL RING
}

\author{
ULI WALTHER
}

(Communicated by Wolmer V. Vasconcelos)

\begin{abstract}
We collect some information about the invariants $\lambda_{p, i}(A)$ of a commutative local ring $A$ containing a field introduced by G. Lyubeznik in 1993 (Finiteness properties of local cohomology modules, Invent. Math. 113, 41$55)$. We treat the cases $\operatorname{dim}(A)$ equal to zero, one and two, thereby answering in the negative a question raised in Lyubeznik's paper. In fact, we will show that $\lambda_{p, i}(A)$ has in the two-dimensional case a topological interpretation.
\end{abstract}

\section{INTRODUCTION}

Throughout let $k$ be a field and let $A$ be a local $k$-algebra. It is shown in [4] that if $A$ is the quotient of a regular local ring $(R, \mathfrak{m}, k)$ of dimension $n$ containing $k, \phi: R \rightarrow A$, ker $\phi=I$, then the Bass number $\lambda_{p, i}(A)=\mu_{p}\left(\mathfrak{m}, H_{I}^{n-i}(R)\right)=$ $\operatorname{dim}_{k} \operatorname{Ext}_{R}^{p}\left(k, H_{I}^{n-i}(R)\right)$ is finite and a function of $A, i, p$ alone but not of $R$ or $\phi$.

Only little is known about the $\lambda_{p, i}$ so far, but they carry interesting information. For example, if $R=\mathbb{C}\left[x_{0}, \ldots, x_{n}\right], \hat{R}=\mathbb{C}\left[\left[x_{0}, \ldots, x_{n}\right]\right]$ and $I \subseteq R$ is the defining ideal of a smooth variety $V \subseteq \mathbb{P}_{\mathbb{C}}^{n}$, then, for $i<n-\operatorname{codim}(V), \lambda_{0, i}(\hat{R} / I \cdot \hat{R})=$ $\operatorname{dim}_{\mathbb{C}}\left(H_{x}^{i}(\tilde{V}, \mathbb{C})\right)$ where $H_{x}^{i}(\tilde{V}, \mathbb{C})$ stands for the $i$-th singular cohomology group of the affine cone $\tilde{V}$ over $V$ with support in the vertex $x$ of $\tilde{V}$ and with coefficients in $\mathbb{C}$.

Since completion does not change $\lambda_{p, i}(A)$ (4, Lemma 4.2) one may assume that $R=k\left[\left[x_{1}, \ldots, x_{n}\right]\right]$. As $H_{I}^{0}(-)=H_{\sqrt{I}}^{0}(-), \lambda_{p, i}(A)=\lambda_{p, i}\left(A_{\text {red }}\right)$. Hence we assume that $I$ is radical. One has $H_{I}^{n-i}(R)=0$ for $i>\operatorname{dim}(A)$ and $\lambda_{p, i}(A)=0$ for $p>i$ by [4], (4.4i) and (4.4ii).

We define the type of the ring $A=R / I$ to be the matrix $\Lambda(A)$ where $\Lambda(A)_{i, j}=$ $\lambda_{i, j}(A)$ for $0 \leq i, j \leq n$.

Recall the Hartshorne-Lichtenbaum vanishing theorem ([2], Theorem 3.1) which we denote by HLVT and in essence states that $H_{I}^{n}(R)=0$ if and only if $I$ is not $\mathfrak{m}$-primary. As is well known, $H_{\mathfrak{m}}^{n}(R)=E_{R}(k)$, the $R$-injective hull of $k$.

Note that by virtue of the spectral sequence

$$
E_{2}^{p q}=H_{\mathfrak{m}}^{p}\left(H_{I}^{q}(R)\right) \Rightarrow E_{\infty}^{p q}=H_{\mathfrak{m}}^{p+q}(R)
$$

Received by the editors June 3, 1999 and, in revised form, September 28, 1999.

2000 Mathematics Subject Classification. Primary 13D45, 14B15; Secondary 14F40.

Key words and phrases. Local cohomology, Lyubeznik numbers.

The author was supported by the A.P. Sloan Foundation. 
and HLVT we have $\Lambda(A)=(1)$ if $A$ is Artinian, and $\Lambda(A)=\left(\begin{array}{ll}0 & 0 \\ 0 & 1\end{array}\right)$ if $\operatorname{dim}(A)=$ 1.

G. Lyubeznik asked in [4] whether $\lambda_{d, d}(A)=1$ for any $A$ and proved it to be true for $A$ normal. We shall show that this is not the case in general.

\section{Pure dimension two}

We shall assume that $k$ is separably closed. This means that in $R$ we can use the second vanishing theorem, due to Ogus, Hartshorne-Speiser and Huneke-Lyubeznik (see [3], Theorem 1.1.): for $\sqrt{I} \subsetneq \mathfrak{m}$, we have $H_{I}^{n-1}(R)=0$ if and only if the punctured spectrum of $R / I$ is connected.

Lemma 2.1. Let $I=\bigcap_{1}^{s} P_{i}$ such that $V(I) \backslash\{\mathfrak{m}\}$ is connected and all $P_{i}$ are prime ideals of dimension 2. Then $H_{I}^{n-1}(R)=0$ and $I$ is of type $\left(\begin{array}{lll}0 & 0 & 0 \\ 0 & 0 & 0 \\ 0 & 0 & 1\end{array}\right)$.

Proof. The second vanishing theorem shows that $H_{I}^{n-1}(R)=0$. The lemma follows from the spectral sequence (1.1).

Proposition 2.2. Let I be radical of pure dimension 2. Let a be the number of connected components of the punctured spectrum of $R / I$. Then $I$ is of type $\left(\begin{array}{ccc}0 & a-1 & 0 \\ 0 & 0 & 0 \\ 0 & 0 & a\end{array}\right)$ and $H_{I}^{n-1}(R)=E_{R}(k)^{a-1}$

Proof. If $a=1$, the claim follows from the previous lemma. If $a>1$, write $I=$ $\bigcap_{1}^{a} J_{k}$ where each $J_{k}$ is radical and defines a connected component of $\operatorname{Spec}(R / I) \backslash$ $\{\mathfrak{m}\}$. Set $J=\bigcap_{1}^{a-1} J_{k}$. By induction, $\lambda_{2,2}(R / J)=a-1$ and $\lambda_{2,2}\left(R / J_{a}\right)=1$. Since $\mathfrak{m}$ is minimal to $J+J_{a}, H_{J+J_{a}}^{n-1}(R)=H_{J+J_{a}}^{n-2}(R)=0$. Hence by the Mayer-Vietoris sequence to $J$ and $J_{a}, H_{J J_{a}}^{n-2}(R)=H_{J}^{n-2}(R) \oplus H_{J_{a}}^{n-2}(R)$ so that $\lambda_{2,2}(R / I)=a-1+1$. Moreover, the Mayer-Vietoris sequence to $J$ and $J_{a}$ also contains a piece

$$
0 \rightarrow H_{J}^{n-1}(R) \oplus H_{J_{a}}^{n-1}(R) \rightarrow H_{J J_{a}}^{n-1}(R) \rightarrow H_{J+J_{a}}^{n}(R) \rightarrow 0
$$

where the last zero comes from HLVT. By induction, the term on the left is isomorphic to $E_{R}(k)^{a-1}$ and in particular injective. The sequence splits and the proposition follows.

\section{The MIXED CASE}

Let $I=J_{1} \cap J_{2}$ where each $J_{i}$ is radical and of pure dimension $i$, and let $a$ be the number of connected components of $\operatorname{Spec}\left(R / J_{2}\right) \backslash\{\mathfrak{m}\}$. Let $x \in J_{2} \backslash \bigcup\{P \mid P \in$ $\operatorname{ass}(I), \operatorname{dim}(P)=1\}$. Then $\operatorname{rad}(I+R \cdot x)=J_{2}$. Consider the long exact sequence of Proposition 8.1.2 in [1]:

$$
\begin{aligned}
0 \rightarrow H_{J_{2}}^{n-2}(R) & \rightarrow H_{I}^{n-2}(R) \rightarrow\left(H_{I}^{n-2}(R)\right)_{x} \rightarrow \\
& H_{J_{2}}^{n-1}(R) \rightarrow H_{I}^{n-1}(R) \rightarrow\left(H_{I}^{n-1}(R)\right)_{x} \rightarrow H_{J_{2}}^{n}(R)=0
\end{aligned}
$$

where the zero on the right comes from HLVT. By [4] (4.4iii), the inclusion $H_{J_{2}}^{n-2}(R)$ $\rightarrow H_{I}^{n-2}(R)$ is an isomorphism. Hence $\lambda_{2,2}(R / I)=\lambda_{2,2}\left(J_{2}\right)$ and we have a four piece exact sequence

$$
0 \rightarrow\left(H_{I}^{n-2}(R)\right)_{x} \rightarrow H_{J_{2}}^{n-1}(R) \rightarrow H_{I}^{n-1}(R) \rightarrow\left(H_{I}^{n-1}(R)\right)_{x} \rightarrow 0
$$


Note that if $M$ is an $R$-module and $x \in \mathfrak{m}$, then $\operatorname{Ext}_{R}^{i}\left(k, M_{x}\right)=0$ for all $i$. Let $F$ be the kernel of the map $H_{I}^{n-1}(R) \rightarrow\left(H_{I}^{n-1}(R)\right)_{x}$ and split the sequence into two short exact sequences. Since $a$ is the number of connected components of the punctured spectrum of $R / J_{2}$, application of $\operatorname{Ext}_{R}^{\bullet}(k,-)$ to the first sequence yields

$$
\begin{aligned}
0 \rightarrow 0 & \rightarrow k^{a-1} \rightarrow \operatorname{Ext}_{R}^{0}(k, F) \rightarrow \\
0 & \rightarrow 0 \rightarrow \operatorname{Ext}_{R}^{1}(k, F) \rightarrow \cdots
\end{aligned}
$$

according to Proposition 2.2. Hence $\operatorname{Ext}_{R}^{0}(k, F)=k^{a-1}$ and $\operatorname{Ext}_{R}^{i}(k, F)=0$ for $i>0$. Application of $\operatorname{Ext}_{R}^{\bullet}(k,-)$ to the second sequence then yields

$$
\begin{aligned}
0 \rightarrow & k^{a-1} \rightarrow k^{\lambda_{0,1}(R / I)} \rightarrow 0 \rightarrow \\
& 0 \rightarrow k^{\lambda_{1,1}(R / I)} \rightarrow 0 \rightarrow \cdots .
\end{aligned}
$$

This proves that $\lambda_{1,1}(R / I)=0, \lambda_{0,1}(R / I)=a-1$ and the type of $I$ equals the type of $J_{2}$.

We present our conclusions in form of the following

Proposition 3.1. Let I be a radical two-dimensional mixed ideal of the complete regular ring $(R, \mathfrak{m}, k)$ where $k$ is separably closed. Write $I=J_{1} \cap J_{2}$ where each $J_{i}$ is radical of pure dimension $i$. Let a be the number of connected components of $\operatorname{Spec}\left(R / J_{2}\right) \backslash\{\mathfrak{m}\}$. Then $I$ is of type $\left(\begin{array}{ccc}0 & a-1 & 0 \\ 0 & 0 & 0 \\ 0 & 0 & a\end{array}\right)$. In particular, the type is independent of the one-dimensional components of $I$.

Remark 3.2. We are not aware of results computing the type of $A$ for general $I$ if $\operatorname{dim}(A)>2$. However, there are some results that relate to the invariants $\lambda_{p, i}(A)$. Known to us are the following:

In [5], the author gives a combinatorial algorithm to calculate the $\lambda_{p, i}(A)$ from a primary decomposition of $I$ assuming that $I$ is a monomial ideal.

In [8] the $\lambda_{p, i}(A)$ for monomial $I$ are investigated in relation to certain Extmodules. Related results have been obtained in [6], where certain combinatorial properties of $H_{I}^{i}(R)$ are studied in the monomial case.

In [7] an algorithm is explained that computes the local cohomology modules $H_{J}^{i}(S)$ if $S$ is a ring of polynomials over a field of characteristic zero, and an algorithm to compute their Bass numbers with respect to a maximal ideal. In particular, the $\lambda_{p, i}(A)$ are computable if $A$ is a quotient of $S$. However, these algorithms do not shed light on structural information about local cohomology in general.

\section{REFERENCES}

[1] M. Brodmann and R. Sharp. Local Cohomology, an algebraic introduction with geometric applications. Cambridge studies in advanced mathematics, 60. Cambridge University Press, 1998. MR 99h:13020

[2] R. Hartshorne. Cohomological dimension of algebraic varieties. Ann. Math., 88:403-450, 1968. MR 38:1103

[3] C. Huneke and G. Lyubeznik. On the vanishing of local cohomology modules. Invent. Math., 102:73-93, 1990. MR 91i:13020

[4] G. Lyubeznik. Finiteness properties of local cohomology modules. Invent. Math., 113:41-55, 1993. MR 94e:13032

[5] J. Montaner. Characteristic cycles of local cohomology modules. JPAA, 150:1-25, 2000.

[6] M. Mustaţă. Local cohomology at monomial ideals. Preprint, 1998. 
[7] U. Walther. Algorithmic Computation of Local Cohomology Modules and the Cohomological Dimension of Algebraic Varieties. J. Pure Appl. Alg., 139:303-321, 1999. CMP 99:16

[8] K. Yanagawa. Bass numbers of local cohomology modules with supports in monomial ideals. Preprint, 1999.

Mathematical Sciences Research Institute, 1000 Centennial Drive, Berkeley, CaliFORNIA 94720

E-mail address: walther@msri.org

Current address: Department of Mathematics, 1395 Mathematical Sciences Building, Purdue University, West Lafayette, Indiana 47907

E-mail address: walther@math.purdue.edu 